\title{
The Mariner 10 Pictures of Mercury: An Overview
}

\author{
Bruce C. Murray \\ California Institute of Technology, Pasadena, California 91125
}

\begin{abstract}
The 11 papers in this issue, comprising the Mariner 10 imaging team final report, are brought together, and the salient results of the Mariner 10 imaging experiment at Mercury are summarized. Those aspects of the data set acquired which were worked by the team are identified, and olher areas where further work is needed are designated.
\end{abstract}

Because of its nearness to the sun as seen from the earth, Mercury has been the least investigated terrestrial planet from earth-based observatories. The flybys of Mariner 10 on March 29 and September 21, 1974, have produced an enormous increase in knowledge. As can be seen in Figure 1, Mariner 10 has placed Mercury in a photographic status similar to that of the moon in the early 1960's, before space exploration began, and similar to the photographic knowledge of Mars since Mariner 9.

A total of 1673 independently useful frames were acquired of Mercury in the first encounter and 540 in the second, most of which are available from the National Space Science Data Center in Greenbelt, Maryland. Preliminary descriptions and interpretations of the photographic data have been published [Murray et al., 1974a, b]. The present set of 11 papers is intended to serve as a final report for the Mariner 10 imaging team, although some additional related papers are expected to be completed in the coming months. In addition, production of airbrush maps of the photographed portion of the mercurian surface is under way in conjunction with the U.S. Geological Survey [see Davies and Batson, 1975], and large orthographically rectified mosaics are under preparation at the Jet Propulsion Laboratory (JPL). These will also be available in reduced form through the National Space Science Data Center. An atlas of the Mariner 10 pictures is also in preparation, under the overall direction of Merton Davies.

It is a useful (and rare) circumstance to be able to compare what has actually been found in the exploration of a new planet with what was anticipated. The two dominant facts about Mercury available before the flight of Mariner 10 were that (l) the bulk density of the planet is $5.45 \mathrm{~g} / \mathrm{cm}^{\mathrm{a}}$, much higher than that of the moon or Mars and quite similar to that of the earth and Venus, and (2) the surface reflection of visible and radio waves, as well as the thermal emission at infrared and radio wavelengths, closely simulates that to be expected from the moon if it were likewise located at the orbit of Mercury. The first fact implies that Mercury as a planet must have a very much higher proportion of iron than Mars or the moon. Indeed, taking into account the self-compression of materials at high pressure in the interior of the earth, the conclusion had been drawn earlier that Mercury must have a somewhat higher percentage of iron than even the earth [Kaula, 1968; Reynolds and Summers, 1969]. Whether the planet was composed of a homogeneous mixture of iron and silicate phase or instead had been differentiated into a large iron core similar to that of the earth with a thin silicate mantle could not be determined from pre-Mariner 10 observations. The second fact, the telescopic similarity to the moon, means that at least the uppermost cen-

Copyright (C) 1975 by the American Geophysical Union. timeters to meters of the surface materials of Mercury on the average are composed of polarized silicates roughly similar to the moon and that the planet has at present very little or no atmosphere (less than around $1 \mathrm{mb}$ ).

On the basis of these meager and disparate facts about Mercury, as well as on extensive recent experience with close-up photography of Mars and the moon, a set of experimental objectives for a flyby imaging experiment were identified [Murray et al., 1971]:

A first objective is to map and idertify the major physiographic provinces on the basis of topographic forms and albedo variations. All of the following questions can be answered by an imaging experiment. What are the similarities and differences between the major surface features of Mercury, the Earth, the Moon, and Mars? Are there impact craters and other exogenic structures? Are there endogenic structures? If mare-like features are present, what is their distribution with reference to the dynamic axis of Mercury, and what is the nature of the mare boundaries? Are there correlations with Earth-based observations, both radar and visual?

At finer scales the topographic form and size distributions of craters can be investigated. Such studies include comparison with slopes and shapes of lunar craters, search for nonlunar cratering processes, variations in crater distributions with latitude and longitude, and estimation of the age of the surface. Evidence of a possible early atmosphere can be sought.

Using photogrammetric techniques, planetary radii at specific points can be compuled and thus it may be possible to determine whether Mercury departs significantly from a spherical shape. By following features across the disk as Mercury rotates, the orientatıon of the spin axis may be able to be specified with greater precision than can currently be determined from Earth. These results can then be combined with images of surface features to establish a coordinate system.

The following additional phenomena can be searched for: features which show peculiar albedo and photometric functions such as rayed craters; indications of radiation darkening and its relationship to that of the Moon and the Galilean satellites; evidences of transitory frosts in the terminator regions; regional color differences, such as are present in the lunar maria.

Due to the extraordinary skill of the engineering groups from JPL and the Boeing Company (prime spacecraft con(ractor), it has been possible to pursue all these experimental objectives in satisfactory detail, in some cases far exceeding our original expectations (except for determination of spin axis orientation). Mercury has been found probably to be a differentiated planet with a large earthlike iron core and, rather surprisingly, to exhibit a surface history closely similar to that recorded on the moon. There are significant implications to the histories of the other terrestrial planets from this lunarlike external record and probably terrestriallike interior of Mercury.

In the sequence of 10 papers that follow are presented the principal findings of the Mariner 10 television team through 

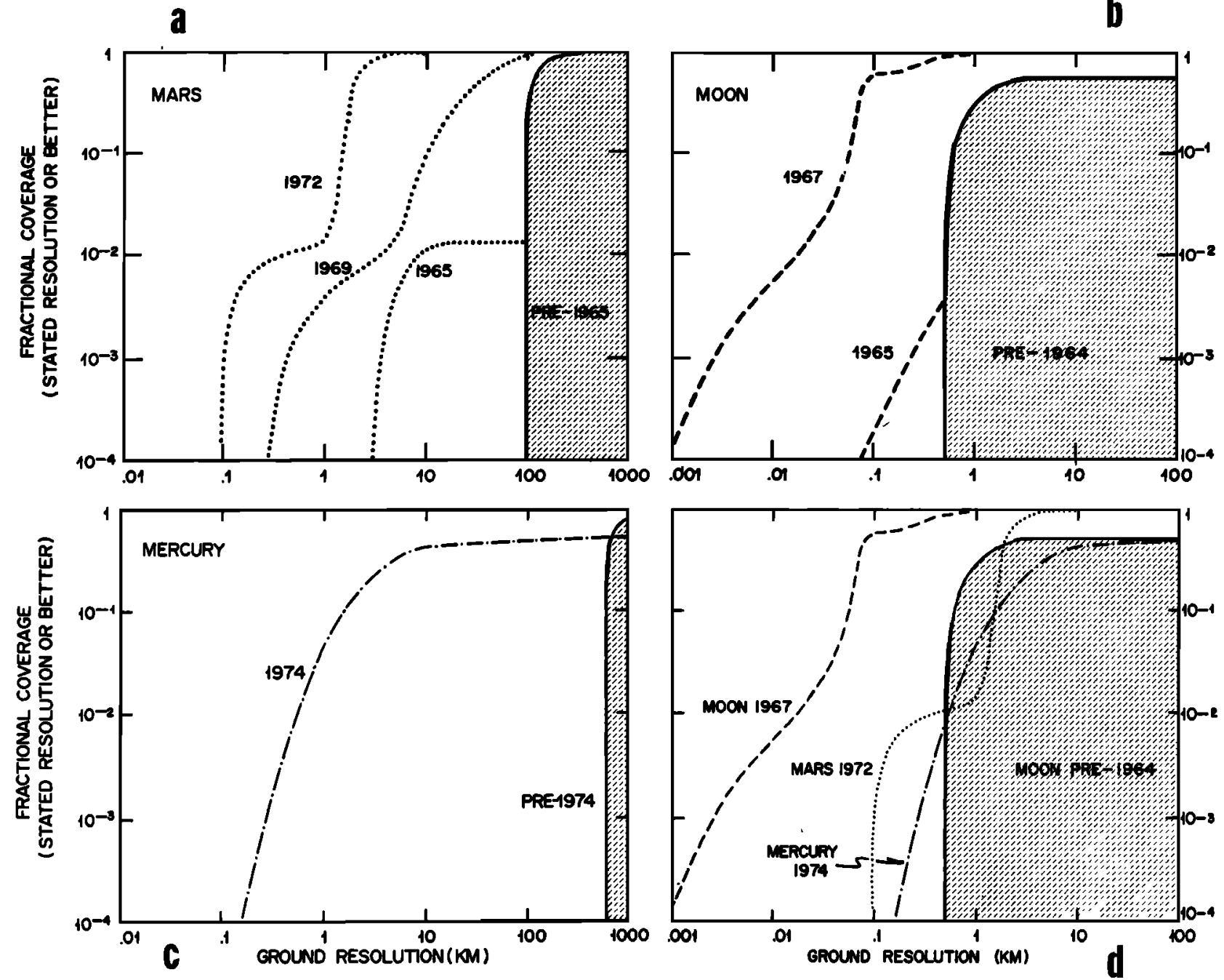

Fig. 1. Resolution versus coverage: All curves in the four parts of Figure 1 are derived by the same guidelines. These include (1) cumulative fractional coverage as a function of surface resolution, (2) elimination of redundant coverage of the same location, and (3) adjustment of resolution for foreshortening. See Murray et al. [1971] and Davies and Murray [1971] for details. (a) Mars imaging coverage versus resolution plots for pre-1965 earth-based results (indicated by stippling), 1965 extension by Mariner 4, 1969 increase by Mariners 6 and 7, and 1972 increase by Mariner 9. (b) Lunar imaging coverage versus resolution plots for pre-1964 earth-based results (indicated by stippling), 1965 extension by Ranger, and 1967 increase due to lunar orbiter coverage. The immensely successful lunar orbiter series of spacecraft photographed selected sites on the lunar near side at resolutions to $1 \mathrm{~m}$ and then proceeded to produce low- and medium-resolution photography of both near and far side to extend lunar coverage to $100 \%$; almost all of the results are at least an order of magnitude better than ground-based results. (c) Mercury photographic coverage resulting from the first and second encounters of Mariner 10 as compared with previous ground-based results. (d) Coverage versus resolution plots showing status of the moon, Mars, and Mercury compared to pre-space age coverage of the moon (indicated by stippling). It can be seen that present coverage and resolution of Mars and Mercury are roughly comparable to the coverage and resolution of the moon obtainable before the space age began.

the analysis of pictures from both the first (Mercury 1) and second (Mercury 2) Mercury encounters.

The Mercury 2 results are presented for the first time in the immediately following paper by Strom et al. [1975a], in which it is shown that the Mercury 2 pictures tend to reinforce the conclusions developed after the first encounter and, especially, emphasize the uniqueness of peculiar hilly and lineated terrains that were observed in the Mercury 1 pictures. The ubiquity of lobate scarps in the terrains exhibiting large impact craters is also supported. The next two papers, 'Acquisition and Description of the Mariner 10 Television Science Data' by Danielson et al. [1975] and 'IPL Processing of Mariner 10 Images of Mercury' by Soha et al. [1975], provide essential background information for all who wish to pursue the study of the Mercury data. Danielson et al. also discuss the results of a preliminary search for any satellites of Mercury, stating 5km diameter as the current upper limit. These papers constitute a valuable record of the techniques and priorities applied in this mission and thus may be relevant to some future space missions as well.

The paper by Klaasen [1975] on 'Mercurian Rotation Period Determined From Mariner 10 Photography' exploits the fact that the heliocentric period of the Mariner 10 spacecraft is commensurate with every two revolutions of Mercury about the sun; in addition, Mercury exhibits a $\% / 2$ commensurability between its axial spin and orbital revolution. As a consequence, identical illumination is encountered by Mariner 10 on each passage. Klaasen shows that limits can be placed upon 
any departure from commensurability of the rotation rate of the planet based on comparison of shadow positions of features contained in overlapping high-resolution pictures. In 'Surface Coordinates and Cartography of Mercury,' Davies and Batson [1975] present both the technique for and the preliminary results of a surface control net and discuss its application to the production of cartographic products. These techniques represent a substantial evolution of those developed previously for the Mariner Mars missions. The current status of nomenclature is also reviewed.

Photometric measurements of Mercury are discussed next by Hapke et al. [1975], taking advantage of what has proved to be better than expected photometric stability of the Mariner 10 vidicon cameras. The photometry, colorimetry, and polarization results show a general similarity to those of the moon, but the authors are able to distinguish certain departures from the lunar color and albedo relationships. Hapke et al. speculate that these mercurian optical properties may arise from small but significant compositional differences from the materials that make up the surface of the moon. Gault et al. [1975] not only describe the morphology of the mercurian impact craters but show significant variations in form from those of the moon. They argue persuasively that these differences reflect primarily the higher surface gravity on Mercury as compared to the moon.

A preliminary geologic terrain map of Mercury has been prepared by Trask and Guest [1975] and is described in their paper. This is an essential step for unravelling the major historical events of the surface as well as providing a basis for future geologic mapping. The crucial geologic issues of the nature of tectonism and the existence of volcanism on Mercury are then discussed by Strom et al. [1975b]. They argue that Mercury exhibits a tectonic style distinct from the moon or Mars; probable compressional features are manifested in the older terrains of Mercury, testifying to an early period of crustal shortening. They also address the question of the origin of the smooth plains deposits and conclude that at least some of the mercurian plains very likely are of volcanic origin. That conclusion carries significant implications for the igneous history of the planet. Finally, in the last paper, by Murray et al. [1975], 'Surface History of Mercury: Implications for Terrestrial Planets,' a working hypothesis is presented for the history of Mercury, drawing upon the previous papers as well as their preferences among many competing theories of planetary history. Then the implications of that 'straw man' history for other terrestrial planets are developed.

A substantial amount of analysis and processing of the Mercury pictures still continues under the auspices of the Mariner 10 television team. Novel rectification procedures have recently been developed to permit stereo viewing of certain portions of the surface; this provides a powerful new tool for photogeologic analysis of Mercury. Other kinds of basic data compilation and analysis are also under way, including presentations of crater frequency curves developed from orthographically projected pictures of the surface, detailed color mapping of the plains deposits, and a refined upper limit for the size of any hypothetical satellites. In addition, the Mercury 3 results can be expected to contribute to further analysis of the surface history and materials. More generally, detailed photogeologic mapping and many topical analyses can be expected to develop as the large community of scientists ex- perienced in the study of the surfaces of the moon and Mars, especially, interact directly with the data themselves. Certainly the broad, rather far-reaching interpretations presented in the last paper in this series will trigger vigorous discussion and expressions of alternate points of view. In so doing, an important principle of planetary exploration may well be illustrated; that is, each new planetary object studied not only adds information significant to its own history and nature but interacts with and accentuates earlier information obtained about other planets. Truly, planetary exploration is a nonlinear process. The opportunity to add a whole new planet to our base of knowledge about the terrestrial planets has been of extraordinary importance, the intellectual implications of which will continue to develop over succeeding years.

Acknowledgments. The cumulative coverage curves of Figure 1 were prepared by James Anderson of the California Institute of Technology who has contributed significantly to the Mariner 10 imaging experiment from inception to conclusion. Contribution 2578, Division of Geological and Planetry Sciences, California Institute of Technology, Pasadena, California 91125.

\section{REFERENCES}

Danielson, G. E., Jr., K. P. Klaasen, and J. L. Anderson, Acquisition and description of Mariner 10 television science data at Mercury, $J$. Geophys. Res., 80, this issue, 1975.

Davies, M. E., and R. M. Batson, Surface coordinates and cartography of Mercury, J. Geophys. Res., 80, this issue, 1975.

Davies, M. E., and B. C. Murray, The View from Space, Columbia University Press, New York, 1971.

Gault, D. E., J. E. Guest, J. B. Murray, D. Dzurisin, and M. C. Malin, Some comparisions of impact craters on Mercury and the moon, $J$. Geophys. Res., 80, this issue, 1975.

Hapke, B., G. E. Danielson, Jr., K. Klausen, and L. Wilson, Photometric observations of Mercury from Mariner 10, J. Geophys. Res., 80, this issue, 1975.

Kaula, W. M., An Introduction to Planetary Physics: The Terrestrial Planets, John Wiley, New York, 1968.

Klaasen, K. P., Mercury rotation period determined from Mariner 10 photography, J. Geophys. Res., 80, this issue, 1975.

Murray, B. C., M. J. S. Belton, G. E. Danielson, M. E. Davies, G. P. Kuiper, B. T. O'Leary, V. E. Suomi, and N. J. Trask, Imaging of Mercury and Venus from a flyby, Icarus, 15, 153, 1971.

Murray, B. C., M. J. S. Belton, G. E. Danielson, M. E. Davies, B. Hapke, B. T. O'Leary, R. G. Strom, V. E. Suomi, and N. J. Trask, Mariner 10 pictures of Mercury: First results, Science, 184, 459, $1974 a$.

Murray, B. C., M. J. S. Belton, G. E. Danielson, M. E. Davies, B. Hapke, B. T. O'Leary, R. G. Strom, V. E. Suomi, and N. J. Trask, Mercury's surface: Preliminary description and interpretation from Mariner 10 pictures, Science, 185, 169, 19746.

Murray, B. C., R. G. Strom, N. J. Trask, and D. E. Gault, Surface history of Mercury: Implications for terrestrial planets, $J$. Geophys. Res., 80, this issue, 1975.

Reynolds, R. T., and A. L. Summers, Calculations on the composition of the terrestrial planets, J. Geophys. Res., 74, 2494, 1969.

Soha, J. M., D. J. Lynn, J. J. Lorre, J. A. Mosher, N. N. Thayer, D. A. Elliot, W. D. Benton, and R. E. Dewar, IPL processing of the Mariner 10 images of Mercury, J. Geophys. Res., 80, this issue, 1975.

Strom, R. G., B. C. Murray, M. J. S. Belton, G. E. Danielson, M. E. Davies, D. E. Gault, B. Hapke, B. O'Leary, N. Trask, J. E. Guest, J. Anderson, and K. Klaasen, Preliminary imaging results from the second Mercury encounter, $J$. Geophys. Res., 80 , this issue, $1975 a$.

R. G. Strom, N. J. Trask, and J. E. Guest, Tectonism and volcanism on Mercury, J. Geophys. Res., 80, this issue, $1975 b$.

Trask, N. J., and J. E. Guest, Preliminary geologic terrain map of Mercury, J. Geophys. Res., 80, this issue, 1975.

(Received February 21, 1975; revised March 3, 1975; accepted March 3, 1975.) 